\title{
Erratum: "Trapping of Plasmons in Ion Holes" [JETP Lett. 77 (12), 647 (2003)]
}

\section{P. K. Shukla and B. Eliasson}

PACS numbers: 52.35.Hr

Equation (6) should read

$$
\begin{gathered}
\tau \frac{\partial^{2} \phi}{\partial \xi^{2}}-\exp \left[\tau\left(\phi-W^{2}\right)\right]+b \exp \left(-\frac{M^{2}}{2}\right) \\
\left.\times\left\{I\left[-\left(\phi-\phi_{\max }\right)\right]+K\left(\frac{M^{2}}{2},-\left(\phi-\phi_{\max }\right)\right)+\frac{2}{\sqrt{\pi|\alpha|}} W_{D}\left[\sqrt{\alpha\left(\phi-\phi_{\max }\right.}\right)\right]\right\}=0 .
\end{gathered}
$$

Equation (7) should read

$$
\begin{gathered}
H(W, \phi, \lambda, M)=3\left(\frac{\partial W}{\partial \xi}\right)^{2}-\frac{\tau}{2}\left(\frac{\partial \phi}{\partial \xi}\right)^{2}-(\lambda-1) W^{2}+\frac{1}{\tau}\left\{\exp \left[\tau\left(\phi-W^{2}\right)\right]-1\right\} \\
+b \exp \left(-\frac{M^{2}}{2}\right)\left[P\left(\phi_{\max }-\phi, \alpha\right)+h\left(\frac{M^{2}}{2}, 0, \phi_{\max }-\phi\right)-1\right]-H_{0}=0 .
\end{gathered}
$$

On page 649 , second column, second paragraph, the expression $3\left(d^{2} W / d \xi^{2}\right)+[1-\exp (\phi)-\lambda] W=0$ should be replaced by $3\left(d^{2} W / d \xi^{2}\right)+[1-\exp (\tau \phi)-\lambda] W=0$. 